\title{
COMPARATIVE ANALYSIS OF PREOPERATIVE AND POSTOPERATIVE DIAGNOSIS IN SKIN MELANOMA
}

\section{Noveski Lazo, Dzonov Boro, Mirchevska Elizabeta, Peneva Margarita, Gruev Vladislav, Dobrevska Jasmina}

University Clinic for Plastic and Reconstructive Surgery, Faculty of Medicine, Ss. Cyril and Methodius University in Skopje, R. North Macedonia e-mail:lazo_noveski@yahoo.com

\begin{abstract}
Introduction: Taking into account the increase in incidence and high mortality in advanced stages, early diagnosis is a prerequisite for successful treatment of melanoma.

The aim of this study was to determine the concordance between dermatological (with dermoscopy), surgical and pathohistological diagnosis in patients with a suspicious finding of skin melanoma.

Material and methods: A comparison between the clinical and pathohistological diagnosis of patients of both sexes, of all ages, with a referral diagnosis of skin melanoma was made.

Results: Out of a total of 535 analyzed cases, 469 (87.5\%) had preoperative dermatological findings. In 329 of these patients the referral diagnosis was in favor of melanoma. In 140 cases with a dermatological finding other than melanoma, a pathohistological finding of melanoma was obtained, $30 \%$ with a dermatological finding.

In addition to melanoma, in 289 cases out of 329 cases with dermatological findings a pathohistological finding for melanoma was obtained, and in 40 cases a pathohistological finding other than melanoma was obtained.

Of 66 cases without dermatological findings, 27 patients with a surgical diagnosis other than melanoma were operated on, with a pathohistological finding for melanoma $-41 \%$. With a diagnosis of melanoma without prior dermatological examination, 39 cases were operated on, of which in 12 cases the pathohistological finding was in support of melanoma.

Of 58 patients who underwent dermoscopy, $78 \%$ had a matching dermoscopic and pathohistological diagnosis. rative diagnoses.

Conclusion: The results of this study showed a high percentage of erroneous preope-

Keywords: melanoma, dermoscopy, misdiagnosis, pathohistology

\section{Introduction}

Despite the growing awareness about melanoma among physicians and people, there are still a large number of cases where timely diagnosis is not made and the disease is not recognized. With the increase in the incidence of melanoma, which is the largest compared to all other malignant diseases, physicians have faced challenges in interpreting and classifying medical data regarding diagnosis of cutaneous melanocyte lesions. Modern diagnostic criteria
\end{abstract}


cause increased subjectivity, which on the other hand creates apparent and greater differences in diagnostic findings among physicians when interpreting findings in melanocyte lesions [1].

Melanoma includes a family of tumors with different potential for growth and metastasis. Fortunately, majority of them grow superficially for years before gaining the potential for vertical growth and metastasis. However, about $10 \%$ of confirmed melanomas are fast-growing, with high biological aggressiveness invading the dermis in just a few months, making it less likely for both the patient and the doctor to detect them in an early stage [2-32]. There are data that some nodular melanomas can grow vertically up to $0.5 \mathrm{~mm}$ per month, which means that in 3-4 months they acquire the potential to metastasize [7]. Therefore, when a patient has paid a visit to a doctor due to his suspicion of melanoma, it is very important to perform all diagnostic and therapeutic procedures in optimal time, which in our opinion should not exceed two months.

The aim of this study was to analyze the concordance among dermatological, surgical and pathohistological diagnosis in patients with admission or pathohistological diagnosis of cutaneous melanoma.

\section{Material and methods}

This prospective-retrospective study included patients of both sexes, of all ages, with a referral diagnosis for skin melanoma, who underwent surgery at the University Clinic for Plastic and Reconstructive Surgery in Skopje in the period from 1.1.2016 to 31.12.2020. The study was part of the scientific project "Comparative analysis of preoperative and postoperative diagnosis in skin meanoma" approved by the Ethics Committee of the Faculty of Medicine, Ss. Cyril and Methodius University in Skopje.

In the retrospective part of the study, the data from patients with admission or pathohistological diagnosis of skin melanoma, operated in the period from 1.1.2016 to 30.6.2018 were analyzed. A comparison between clinical and pathohistological diagnosis was made, based on findings that are part of the usual work protocol.

In the prospective part of the study, the findings of the patients operated at the Clinic with admission or pathohistological diagnosis for skin melanoma in the period from 1.7.2018 to 31.12. 2020 were analyzed. In this part of the study it was emphasized that physicians should comply with the existing work protocols.

The concordance between the clinical and pathohistological diagnosis in patients from the retrospective part of the study, the concordance of dermatological, surgical and pathohistological diagnosis in patients from the prospective part of the study, as well as comparison of the results from the retrospective and prospective part of the study were made.

\section{Results}

In the period from 2016 to 2020 at the University Clinic for Plastic and Reconstructive Surgery, a total of 790 patients associated with the diagnosis of melanoma were operated on, of whom 535 patients were included in this study.

In spite of the fact that the physicians were asked to follow the existing protocols, dermoscopy was performed only in 58 patients $(19.7 \%)$ of a total of 294 operated patients in the prospective part of the study. This has indicated non-adherence to the recommendations and work protocols by surgeons during treatment.

Of the 535 analyzed cases, $469(87.5 \%)$ had preoperative dermatological findings. In 329 of these patients, the referral diagnosis went in favor of melanoma. It has to be noted that due to suggestibility, diagnoses such as suspicious for melanoma (melanoma in obs) were also taken into account. In 221 of 329 patients, the referral diagnosis contained multiple 
differential diagnostic entities (usually 2-3), and in 108 cases the referral diagnosis was melanoma only (including 51 with a dermoscopic finding of melanoma).

In 140 cases with a dermatological finding other than melanoma (including 7 with a dermoscopic finding), a pathohistological finding of melanoma was obtained, which means that $30 \%$ of 469 cases with a dermatological finding were false negative.

Out of 329 patients with dermatological findings consistent with melanoma (including 51 cases with dermoscopic findings), in 289 cases a pathohistological finding of melanoma was obtained, and in 40 cases (including 6 cases with dermoscopic findings) a pathohistological finding other than melanoma was obtained, $12 \%$ of 329 (false positive).

The most common dermatological diagnoses other than melanoma at the time of referral to surgical treatment, which were largely accepted by the surgeon at initial treatment, are shown in Table 1.

Table 1. The most common dermatological diagnoses other than melanoma when referring to surgical treatment

\begin{tabular}{lc}
\hline \multicolumn{1}{c}{ Dermatological dg } & Number of cases \\
\hline different types of nevi & 83 \\
carcinoma basocellulare & 21 \\
carcinoma planocellulare & 13 \\
keratosis seborrhoica & 11 \\
haemangioma & 5 \\
keratoacanthoma & 3 \\
granuloma pyogenicum & 2 \\
dermatofibroma & 2 \\
\hline
\end{tabular}

Out of 66 cases without dermatological findings, referred by a family doctor or a doctor of another specialty, with a surgical diagnosis other than melanoma, 27 patients were operated on, with a pathohistological finding of melanoma - $41 \%$ (false negative).

Thirty-nine patients with a diagnosis of melanoma without prior dermatological examination were operated on, of whom in 12 cases the pathohistological finding was consistent with melanoma, and in 27 cases it was different from melanoma - 69\% of 39 cases (false positive).

The fact there was a high percentage $(87.5 \%)$ of cases with a dermatological finding raised suspicions and, hence, we decided to merge the dermatological and surgical findings as a clinical diagnosis.

The results obtained from the analysis of the concordance among dermatological (with and without dermatoscopic findings), surgical and pathohistological diagnosis are shown in Table 2.

Table 2. Matching between clinical and pathohistological diagnosis in melanoma

\begin{tabular}{ccccc}
\hline Year & Correct dg & False negative dg & False positive dg & Total \\
\hline 2016 & $57(62 \%)$ & $26(28 \%)$ & $9(10 \%)$ & 92 \\
2017 & $62(55 \%)$ & $41(37 \%)$ & $9(8 \%)$ & 112 \\
2018 & $56(52 \%)$ & $40(37 \%)$ & $12(11 \%)$ & 108 \\
2019 & $79(58 \%)$ & $38(28 \%)$ & $19(14 \%)$ & 136 \\
2020 & $47(54 \%)$ & $22(25 \%)$ & $18(21 \%)$ & 87 \\
Total & $301(56 \%)$ & $167(31 \%)$ & $67(13 \%)$ & 535 \\
\hline
\end{tabular}

Out of 58 patients who underwent dermoscopy, $78 \%$ had a concordance between the dermoscopic and pathohistological diagnosis (Table 3, Figure 1). 


\begin{tabular}{|l|l|c|}
\hline \multicolumn{3}{|c|}{ Table 3. Misdiagnosis with dermoscopy (of a total of 58) } \\
\hline & Dermoscopic dg & Pathohistological dg \\
\hline 1 & Melanoma & Acanthosis \\
\hline 2 & Melanoma & Ca basocellulare \\
\hline 3 & Melanoma & Poroma \\
\hline 4 & Melanoma & Ca basocellulare \\
\hline 5 & Melanoma & Nevus dysplasticus \\
\hline 6 & Melanoma & Melanoma IIA \\
\hline 7 & Ca basocellulare & Melanoma IA \\
\hline 8 & Nevus pygmentosus & Melanoma IB \\
\hline 9 & Nevus dysplasticus & Melanoma IB \\
\hline 10 & Nevus atipicus & Melanoma IIB \\
\hline 11 & Nevus & Melanoma in situ \\
\hline 12 & Nevus & Melanoma IIB \\
\hline 13 & Dermatofibroma \\
\hline
\end{tabular}

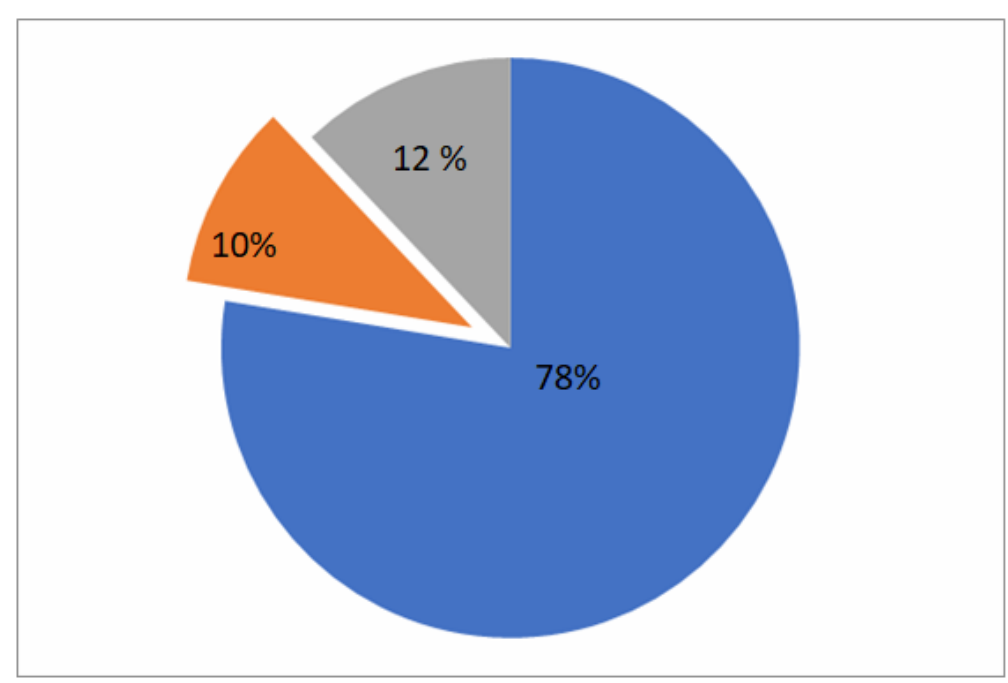

Fig. 1. Matching between dermatoscopic and pathohistological diagnoses

Dermatoscopic diagnosis of melanoma - pathohistological diagnosis different from melanoma (overdiagnosed- false positive)

Dermatoscopic diagnosis different from melanoma - pathohistological diagnosis of melanoma (misdiagnosed- false negative)

Dermatoscopic diagnosis of melanoma - pathohistological diagnosis of melanoma

The results shown in Tables 4, 5 were obtained from the analysis of the coincidence of the clinical (dermatological and surgical) and pathohistological diagnosis.

Table 4. Matching between clinical and pathohistological diagnosis in the retrospective part of the study

\begin{tabular}{lcccc}
\hline Year & Correct dg & false negative dg & false positive dg & Total \\
\hline 2016 & 57 & 26 & 9 & 92 \\
2017 & 62 & 41 & 9 & 112 \\
2018 & 17 & 14 & 6 & 37 \\
Total & 136 & 81 & 24 & 241 \\
\hline
\end{tabular}


Table 5. Coincidence between clinical and pathohistological diagnosis in the prospective part of the study

\begin{tabular}{ccccc}
\hline Year & Correct dg & false negative dg & false positive dg & Total \\
\hline 2018 & 39 & 26 & 6 & 71 \\
2019 & 79 & 38 & 19 & 136 \\
2020 & 47 & 22 & 18 & 87 \\
Total & 165 & 86 & 43 & 294 \\
\hline
\end{tabular}

Out of 468 pathohistologically confirmed melanomas, only in two cases the initial pathohistological finding was different from melanoma, so a diagnosis of melanoma was made upon request for revision.

The mean waiting time for pathohistological findings in primary melanoma was 25.7 days (3-91), and the mean waiting time for pathohistological findings in melanoma metastases was 20.7 days $(8-55)$.

Table 6. Summary of matching diagnoses in relation to surgeons who have worked

\begin{tabular}{lccccc}
\hline Surgeon & Correct dg & $\begin{array}{c}\text { False } \\
\text { negative dg }\end{array}$ & $\begin{array}{c}\text { False positive } \\
\text { dg }\end{array}$ & Total & $\begin{array}{c}\text { Percentage } \\
\text { of error }\end{array}$ \\
\hline 1 & 8 & 3 & 8 & 19 & $57 \%$ \\
2 & 8 & 8 & 1 & 17 & $53 \%$ \\
3 & 22 & 10 & 5 & 37 & $40 \%$ \\
4 & 57 & 18 & 6 & 81 & $30 \%$ \\
5 & 12 & 10 & 5 & 27 & $55 \%$ \\
6 & 21 & 8 & 4 & 33 & $36 \%$ \\
7 & 1 & 1 & 1 & 3 & $67 \%$ \\
8 & 29 & 17 & 6 & 52 & $44 \%$ \\
9 & 20 & 22 & 5 & 47 & $57 \%$ \\
10 & 19 & 17 & 4 & 40 & $52 \%$ \\
11 & 29 & 4 & 3 & 36 & $19 \%$ \\
12 & 13 & 10 & 3 & 26 & $50 \%$ \\
13 & 27 & 15 & 7 & 49 & $45 \%$ \\
14 & 13 & 8 & 2 & 23 & $43 \%$ \\
15 & 22 & 16 & 7 & 45 & $51 \%$ \\
Total & 301 & 167 & 67 & 535 & $44 \%$ \\
\hline
\end{tabular}

The analysis of individual error of surgeons treating melanoma patients was performed, and it showed significant individual differences in performance (Table 6).

\section{Discussion}

Diagnostic errors in the treatment of melanoma have been the subject of analysis in several studies [8-11]. The results of our study do not completely match with the published data. The main difference is that both false positive and false negative diagnoses have been analyzed in this study. In the literature, analyses that involve only false negative findings are common [8-11]. An extremely high percentage (44\%) of diagnostic errors is an alarm for additional analyses and for taking measures to amend the situation. It is obvious that most of the mistakes have been made in the group of false negative diagnoses. We believe that the large number of errors in the diagnosis and treatment has been made by surgeons driven by the desire and intent to remove the threat of the disease as soon as possible. (The large number of false negative diagnoses does not support this view).

Therefore, we want to discuss some analyses and opinions presented in different sources that have studied the topic of diagnostic and therapeutic errors, and to debate over the high percentage of errors in our study. 
According to Newman-Toker et al. a diagnostic error is a failure to provide an accurate and timely explanation of a patient's health problem, or a failure to communicate with a patient about a health problem $[8,9]$. Misdiagnosis is defined as a delay or failure in the treatment of a health condition that is present, where the occupational diagnosis is incorrect or nonexistent (false negative), or as treatment applied to a condition that does not exist (false positive). In his research on errors in several groups of diseases ("Big Three"), false positive cases were not taken into account, so the percentage of false diagnoses (false negative) for melanoma was $13.6 \%$. The study used data from the Queensland Cancer Registry [8, 9].

As early as 2003, Osborne analyzed errors in diagnosing melanoma in 730 cases, depending on the specialty of the doctors who had made the diagnosis. According to his results, the error rate of $29 \%$ was reported for dermatologists, $19 \%$ for plastic surgeons, $54 \%$ for family doctors and $55 \%$ for doctors of other specialties.

The results of the study by Urbancek S. et al. showed that in 936 histologically confirmed melanomas, a misdiagnosis was made in $16 \%$ of patients. False-positive diagnoses were not considered in this study [11].

In her study, Olga Simonescu highlighted the mistakes made in all steps of the diagnostic process and in the treatment of 33 patients with melanoma. It recorded 166 errors in all segments of the process: 36 clinical diagnostic errors, 31 surgical, 24 pathohistological errors, 13 sentinel node biopsy errors, 17 staging errors, and 45 treatment errors.

Unfortunately, very little literature is available today that addresses the issue of errors in the diagnosis of melanoma, as well as the cause of those errors. As early as 1985, Ackermann, long before his time, expressed hope and belief that no man should die of melanoma $[1,6]$. His views were based on the knowledge that pathohistologically melanoma can be diagnosed when it is confined to the epidermis, before invading the dermis (melanoma in situ). But he neglected the question of how melanoma could be clinically recognized and operated on at such an early stage. At that time, melanoma was most often diagnosed when it was already ulcerated or bleeding. The introduction of the ABCD rule in the mid-1980s helped in making clinical diagnosis of melanoma and in urging patients to seek medical help earlier. But even this rule is not enough to recognize and diagnose melanoma with the naked eye at an early stage.

With the introduction of dermoscopy, a revolutionary breakthrough was made in the early diagnosis of melanoma, before it could be diagnosed on the basis of macroscopic (visible to the naked eye) signs and criteria, which created the conditions to realize Ackerman's vision.

But, in reality these expectations do not come true. Although the incidence of earlystage melanoma has increased dramatically in the last decade, the mortality rate for advanced melanoma appears to remain stable. Rousseau attributes the causes of this condition to tumor biology, misconceptions and lack of education in patients, and errors in the approach and treatment made by physicians [1].

The problem with aggressive tumor biology becomes unclear as part of the knowledge and hypotheses about the development of some melanoma from dermal cells, which is different from the conventional concept of melanoma development by the transformation of epidermal melanocytes. An even greater dilemma in these fast-growing melanomas (primarily dermal, purely nodular) is the evidence that they develop in patients with a small number of nevi or without known risk factors. Although they are a small part of all melanomas, they definitely have a high impact on mortality, and that influence seems impossible to be eliminated. This implies that the existing screening strategies will not be successful for such patients, which indicates the need for their supplementation [1]. 
In everyday practice, many patients are diagnosed with melanoma in the advanced stage of the disease, due to lack of awareness or widespread misconception. Piccolo's study of 1,026 adult women in Italy found that $82 \%$ of them believed that excision of existing moles was dangerous, and that the operation itself could cause their malignancy to worsen.

The association between socioeconomic status and stage of melanoma has been reported in several studies showing that low socioeconomic status is associated with a higher stage of melanoma at diagnosis as well as increased mortality [1].

Therefore, screening programs, if not combined with systematic efforts to combat these misconceptions, may be ineffective in raising awareness.

The most likely reason why doctors make mistakes is not their ability to recognize melanoma, but rather they do not give themselves a chance to see and examine it. It is known that a large percentage of melanomas are detected by patients, and the rest of the melanoma should be detected by doctors. Thus, they should clinically examine all pigmented changes in patients, which in most cases is not done. Even dermatologists use a complete skin examination in only $30 \%$ of patients. In practice, it is unrealistic to expect all patients to have a complete skin examination. Therefore, a complete examination is recommended to be mandatory in individuals belonging to high-risk groups. Epidemiological data are well known: high-risk groups include individuals with a history of: 1) skin malignancy, 2) more than 20 nevi on the hand (which is predictive of a high total number of nevi, and 3) sun-damaged skin in uncovered regions [1].

The strategy for a complete body examination is recommended to be applied at the primary level, i.e., by a family doctor, which in some countries gives excellent results.

The introduction of dermoscopy as a method represents a diagnostic revolution in the field of cutaneous malignancies. Unfortunately, very few patients in our study had dermoscopic findings, indicating an incorrect approach to the diagnostic process. Of the patients with dermoscopy (58), 22\% were misdiagnosed, which was far better when compared to the total number of errors made in the remaining patients (44\%). Despite the small number of patients analyzed, the application of dermoscopy has proved to be a superior diagnostic method, indicating the need for its introduction as a mandatory part of the diagnostic process.

Many authors have presented their suggestions for improving dermoscopy performance in order to avoid errors in the findings. According to Kaminsk-Grazina-Vinciorek, the basic errors in dermatoscopy can be divided into errors caused by insufficient knowledge and errors due to the choice of the wrong method (procedural or technical errors) [13].

In our study, out of 468 pathohistologically confirmed melanomas, only in two cases was the initial pathohistological finding different from melanoma; hence, a diagnosis of melanoma was made upon request for revision.

In conclusion, despite all the previously known recommendations and adopted protocols, we have not managed to implement them. According to the results obtained in this study, we believe that when malignant diseases (in this case melanoma) are in question, short-term (annual) analyses are needed by the health institution management and state healthcare authorities in order to timely point out to individual's mistakes and the need of improvement and adherence to certain procedures in the process. Of course, the process itself should include doctors from preventive medicine who should have insight in the statistical data and alert on time about the negligence made in the process, which is not the case in our institution.

Conflict of interest statement. None declared. 


\section{References}

1. Russo T, Lallas A, Brancaccio G, Piccolo V, Alfano R, Argenziano G. No One Should Die of Melanoma: Time for This Vision to Be Realized? Dermatol Pract Concept 2019; 9(1): 1-3.

2. Whiteman DC, Pavan WJ, Bastian BC. The melanomas: a synthesis of epidemiological, clinical, histopathological, genetic, and biological aspects, supporting distinct subtypes, casual pathways, and cells of origin. Pigment Cell Melanoma Res 2011; 24: 879-97.

3. Brancaccio G, Russo T, Lallas A, Moscarella E, Agozzino M, Argenziano G. Melanoma: clinical and dermoscopic diagnosis. G Ital Dermatol Venereol 2017; 152(3): 213-23.

4. Russo T, Piccolo V, Ferrara G, Agozzino M, Alfano R, Longo C, Argenziano G. Dermoscopy pathology correlation in melanoma. J Dermatol 2017; 44(5): 507-14.

5. Simionescu O, Blum A, Grigore M, Costache M, Avram A, Testori A. Learning from mistakes: errors in approaches to melanoma and the urgent need for updated national guidelines. Int J Dermatol 2016; 55(9): 970-6.

6. Greenwald HS, Friedman EB, Osman I. Superficial spreading and nodular melanoma are distinct biological entities: a challenge to the linear progression model. Melanoma Res 2012; 22(1): 1-8.

7. Newman-Toker DE, Wang Z, Zhu Y, Nassery N, Saber Tehrani AS, Schaffer AC, et al. Rate of diagnostic errors and serious misdiagnosis-related harms for major vascular events, infections, and cancers: toward a national incidence estimate using the "Big Three". Diagnosis (Berl) 2020; 8(1): 67-84.

8. Newman-Toker DE, Schaffer AC, Yu-Moe CW, Nassery N, Saber Tehrani AS, Clemens GD, et al. Serious misdiagnosis-related harms in malpractice claims: The "Big Three" - vascular events, infections, and cancers. Diagnosis (Berl) 2019; 6(3): 227-40.

9. Osborne JE, Chave TA, Hutchinson PE. Comparison of diagnostic accuracy for cutaneous malignant melanoma between general dermatology, plastic surgery and pigmented lesion clinics. Br J Dermatol 2003; 148(2): 252-8.

10. Urbancek S, Fedorcova P, Tomkova J, Sutka R. Misdiagnosis of Melanoma: A 7 Year Single-Center Analysis. J Pigment Disord 2015; 2: 208.

11. Piccolo V, Russo T, Giacomel J, Lallas A, Alfano R, Argenziano G. Dispelling myths concerning pigmented skin lesions. J Eur Acad Dermatol Venereol 2016; 30(6): 919-25.

12. Kamińska-Winciorek G, Placek W. The most common mistakes on dermatoscopy of melanocytic lesions. Postepy Dermatol Alergol 2015; 32(1): 33-9. 